\title{
Evaluation of Consumer Acceptability of Meat Processing Methods
}

\author{
T. $\mathrm{Lu}^{1,3}$, P. Wall ${ }^{1,3}$, M. Burke ${ }^{4}$ N. McCaffrey ${ }^{5}$ and A.G.M. Scannell ${ }^{2,3}$ \\ ${ }^{1}$ School of Public Health, Physiotherapy and Sports Science, \\ ${ }^{2}$ School of Agriculture and Food Science, \\ ${ }^{3}$ Institute of Food and Health, University College Dublin, Belfield, Dublin 4, Republic of Ireland, \\ ${ }^{4}$ Devenish Nutrition, Lagan House, 19 Clarendon Rd, Belfast BT1 3BG Northern Ireland and \\ ${ }^{5}$ Ipsos MRBI, Block 3, Blackrock Business Park, Blackrock, Co. Dublin, Republic of Ireland
}

Campylobacter is the commonest cause of bacterial food poisoning on the island of Ireland ${ }^{(1)}$. The prevalence of Campylobacter on retail poultry on the island of Ireland ranges widely, from approximately $50 \%$ to over $90 \%{ }^{(2)}$. Many processing methods have been investigated regarding the efficacy in reducing Campylobacter in raw retail poultry. This study aims to explore consumer acceptance of a number of processing technologies proven to reduce Campylobacter.

During the qualitative research, a total of five consumer focus groups (9 respondents per group) were conducted across ROI and NI, involving a mix of ages and life stages, with two groups female only and three groups mixed gender. Based on the reactions noticed during the groups as well as how the proposed treatments were analyzed by industry experts, the 6 treatments chosen to be analyzed during the subsequent quantitative stage were Crust Freezing, Steam Ultrasound, Forced Air Chilling, Organic Acid Wash, Chemical Wash and Cold Plasma treatment.

In quantitative research, after being given some explanations, forced air chilling $(55 \%)$ ranked as the most acceptable intervention while chemical washes $(16 \%)$ were considered the least acceptable. The data was also analyzed using cluster analysis. $33 \%$ of respondents were indifferent to interventions of any type, $27 \%$ found some acceptable, $21 \%$ found some partially acceptable, $19 \%$ were against every method. The survey established that the public have clear negative views about chemical forms of treatment with more positive views of physical treatments. Given sufficient information, people become more positive about some forms of treatment. However it appeared that the language used in the explanations given to the respondents was not sufficiently convincing for many consumers.

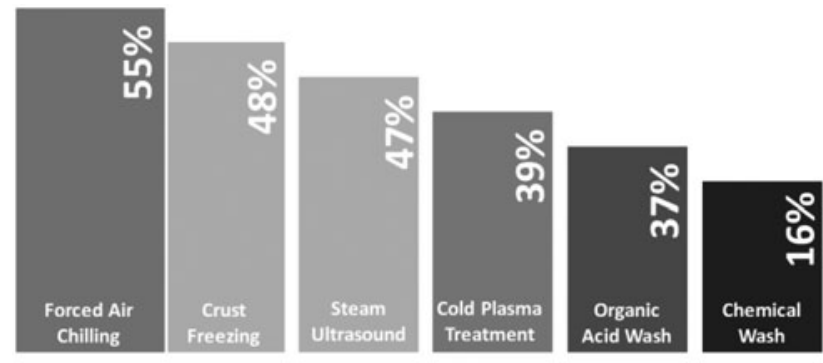

Consumer Acceptability of Meat Processing Methods

This study highlights the gap that exists between the public and professional views of what is acceptable. It highlighted the challenge for communicators framing messages that can effectively reassure consumers that interventions are beneficial. More innovative communication strategies are needed to generate consumer confidence in interventions Science communication requires competencies that many conventional scientists do not have however if scientific developments are to be utilized strategies to encourage consumer confidence and acceptance have to be considered.

This project was generously funded by Safe Food, Republic of Ireland.

1. EFSA, ECDC (2015) The EFSA Journal 11, 3129.

2. Madden RH, Moran L, Scates P, et al. (2011). Journal of food protection 74, 1912-1916. 